\title{
Reconsidering the risk assessment concept: Standardizing the impact description as a building block for vulnerability assessment
}

\section{K. Hollenstein}

Forest Engineering, Swiss Federal Institute of Technology Zurich

Received: 28 September 2004 - Revised: 6 January 2005 - Accepted: 15 March 2005 - Published: 29 March 2005

Part of Special Issue "Multidisciplinary approaches in natural hazard and risk assessment"

\begin{abstract}
Risk assessments for natural hazards are becoming more widely used and accepted. Using an extended definition of risk, it becomes obvious that performant procedures for vulnerability assessments are vital for the success of the risk concept. However, there are large gaps in knowledge about vulnerability. To alleviate the situation, a conceptual extension of the scope of existing and new models is suggested. The basis of the suggested concept is a stadardization of the output of hazard assessments. This is achieved by defining states of the target objects that depend on the impact and at the same time affect the object's performance characteristics. The possible state variables can be related to a limited set of impact descriptors termed generic impact description interface. The concept suggests that both hazard and vulnerability assessment models are developed according to the specification of this interface, thus facilitating modularized risk assessments. Potential problems related to the application of the concept include acceptance issues and the lacking accuracy of transformation of outputs of existing models. Potential applications and simple examples for adapting existing models are briefly discussed.
\end{abstract}

\section{Introduction}

Risk assessments have become increasingly popular for application with natural hazards in Switzerland as well as other countries. They provide an avenue for improving the efficiency of protection measures, since they focus on damage rather than on hazards. However, while important progress has been made in the field of hazard analysis, the other aspects of risk assessments are only poorly developed. To exploit the full potential of the risk concept, it is crucial that it is evolved in a methodologically coherent way and at an equal pace. The author argues that in the future more emphasis should be placed on the research addressing the targets at

Correspondence to: K. Hollenstein

(kurt.hollenstein@env.ethz.ch) risk to ensure the overall quality of risk assessments. Vulnerability analysis is probably the area that is both the least developed and most difficult one to address, requiring that available knowledge and resources are used to their full extent.

\section{Status of vulnerability analysis and current state of} knowledge

The simplest definition of risk is

$R=F \times N$

where $F$ is the frequency of an event occurring and $N$ the damage. This formulation comes from technical risk analyses, and it is originally based on so-called level-1 assessments where the focus is on "in-plant" damage (especially core meltdowns in the nuclear industry). Risk is thus a representation of the expected amount of damage for a given period of time.

For natural hazards, an extension of this concept is useful since there is no such thing as an in-plant perspective. Risk in the context of natural hazards is characterized by hazardrelated aspects on one hand and target-related aspects on the other hand. The hazard is defined via a probability $P$ (or its reciprocal, the return period) and an intensity $I$ (comprising a description of the impact, ideally together with its spatiotemporal distribution). Generally, a relation of the form

$P=f(I)$

holds, and it is often possible to determine an analytic form for $f$ (e.g., extreme value statistics, power laws).

The target side is also characterized by two factors: the exposure $E$ (describing the spatiotemporal distribution of the target objects) and the vulnerability $V$. Thus, for the purposes of natural hazard risk analyses, risk can be defined as

$R=P \times I \times E \times V$ 
Following Eq. (2), it is obvious that vulnerability analysis is a key part of the risk assessment for natural hazards. One would expect that there are proven, well accepted methods and models available for performing vulnerability analyses. However, a State-of-the-Art review showed a different picture Hollenstein et al. (2002). First, there are significant differences in the definition of vulnerability. Broadly, the following definitions werde used:

- Vulnerability as a boolean variable. According to this, the term vulnerability merely reflects whether or not a given object (i.e., a component, structure or system) can be negatively affected by the effects of a certain natural hazard. Within the concept of risk represented by Eq. (2), the use of boolean vulnerability is limited to synoptic assessments and to the identification of objects at risk, but it cannot provide siginficant inputs to detailed and scalable quantitative assessments. This definition of vulnerability was used in rougly $30 \%$ of the literature citatitions considered.

- Vulnerability as a semiquantitative variable. In this case, the vulnerability expresses the degree of the negative effects using pre-defined, mostly qualitative discrete classes such as "no damage", "minor damage", "severe damage" or "total damage". If these classes are consistently defined for all objects (such that the class value can be related to the value of the object's performance characteristics), such a semi-quantitative formulation can usually provide results that are sufficiently accurate for most purposes. $23 \%$ of the literature sources used this definition.

- Vulnerability as a fully quantitative variable. In this case the vulnerability again represents the degree of the negative effects, but not on the basis of qualitatively defined classes, but as absolute or (more common) relative values. While providing (at least in theory) the most accurate information, this formulation is by far not as frequent as the semiquantitative one. To some extent, this is due to the large uncertainties that are inherent in vulnerability assessments for all but the most simple objects.

- Vulnerability as an interpolated variable. In many cases, semi-quantitative vulnerability defintions are transformed into quantitative ones by using characteristic values for the individual classes (e.g., by representing "minor damage" as $0 \%$ to $5 \%$ with an average of $2.5 \%$ as characteristic value) and determining an interpolation function that can be used more efficiently in high-volume computations. A well-known example of this type of vulnerability definition is ATC (1985). This and the previous type accounted for about $40 \%$ of the citations.

Besides that, there are numerous cases where the term vulnerability was used, but without explicitly defining what its meaning is.
[NB: The selection of the literature citations used as a basis for this and other classifications may be subject to errors of omission. This is addressesd in detail in Hollenstein et al. (2002). Due to the scarcity of models for certain harads, reasonable statistical data could only be derived for earthquake models.]

In addition to the definition of vulnerability, and with the intention of judging the cited models for their potential applicability for every-day risk assessments, the following classification criteria were used:

- Subject: the type of natural hazard the model is applied to

- Potential applicability: limited to the specific application domain/applicable to other domains/generic

- Methodology for describing the hazard: case study/statistical description/probablistic description/use of fuzzy sets

- Methodology for describing the target object: case study/statistical description/probablistic description/use of fuzzy sets

- Application field:

engineering disciplines/insurance/government activities

- Target audience: engineering disciplines/insurance/government activities

- Orientation: basic science/practical application

- Input parameters for hazard representation

- Input parameters for target representation

- Output parameters of hazard representation

- Output parameters of target representation

- Distinction between hazard and target modelling: clear separation/obvious distinction between submodels/distinction not obvious, combined hazard-target modelling

- Definition of vulnerability (see above)

With regard to the subject, it was surprising to the author how the number of available models differs between the natural hazards investigated. While there were numerous hits $(>1000)$ when searching for earthquake and wind $(>100)$ related vulnerability models, there were only a few $(<20)$ ones for gravitational hazards such as landslides, debris flows, snow avalanches and even floods. The abundance for the earthquake and wind models may be explained by the economic impacts of these hazards, but the scarcity of models for the other hazards is by far not in agreement with their associated damage. One potential reason is that these gravitationally driven processes are usually delimited quite accurately, and in many cases the approach to managing these 
risks is simply avoiding the areas potentially affected. Consequently, these hazards are not (or at least not explcitly) taken into account in the design of structures, whereas the ubiquitous processes are dealt with. Another potential reason is that there exists sufficient empirical knowledge at the institutions that are responsible for managing these risks, and that there is thus little or no demand for theoretical models. However, the third and most probable explanation is that the issue of vulnerability was in the past simply not addressed on a systematic level for these "minor" hazards. This suggestion is supported by the IUGS in a statement of their working group on landslides which says: Although the state of the art for identifying the elements at risk and their characteristics is relatively well developed, the state of the art for assessment of vulnerability is in general relatively primitive ... IUGS (1997). It seems reasonable to assume that the same could be said for the other gravitational hazards.

When the applicability is considered, roughly $40 \%$ of the models were judged to be applicable only for the hazardtarget combination they were derived from. However, judging $60 \%$ of the models as applicable for other purposes is extermely opimistic and does not account for other than methodological differences (i.e., issues such as data availiability or comparability are not addressed).

Not surprising is the fact that rougly $90 \%$ of the models are practically and only $10 \%$ theoretically oriented. This is largely due to the fact that vulnerabilty is principally something that has to be dealt with in the "real world". Engineering judgements about the sensitivity of given structures is usually not a key topic of science, but a very important issue in everyday life.

For a more detailed analysis of the other criteria, the reader is encouraged to refer to Hollenstein et al. (2002).

\section{A concept for standardizing the scope and basis of vulnerability analysis within risk analysis}

Considering that there are many vulnerability assessment methods available for some hazards and none or only very few ones for other hazards, it would obviously be appealing to apply the existing models beyond their original scope to fill in the gaps. At the same time, new hazard and vulnerability models being developed could be more useful if they were applicable not only for one specific hazard-target combination, but for a whole group of target objects and hazards or even for arbitrary ones. Hazard and vulnerability analysis would then become totally independent form each other.

[This idea was also the rationale for selecting most of the other evaluation criteria in the list shown above: they can be used i) for assessing the suitability of a model for being used for other purposes than originally designed for and ii) for evaluating its (conceptual) potential as a basis for developing "generic" vulnerability analysis methods.]

Whether or not a model can be used for assessing the vulnerability of a certain target with regard to a particular hazard primarily depends on the representation of the input that the model requires, i.e. on the specification of the impact parameters. Our review has shown that about $80 \%$ of the available models are specificically designed for an explicit combination of hazard and target object or derived exclusively from empirical or experimental data (see e.g. Dowrick and Rhoades (1997), Dameron and Parker (1996), Balendra et al. (1999)). They often either use a specific interface between the hazard and the target rather than a generic one or they even lack a clear distinction between hazard and target modelling (i.e., it is not clear which parts of the model describe the target). Ther are a few more generic concepts such as the "parameterless scale of intensity" approach suggested by Coburn and Spence (1992) or the "assembly-based vulnerability" method shown in Porter et al. (2001). It has also been tested to what extent and with what accuracy existing model can be applied to other hazards, as in the case of Dean and Soulage (1999), where wind design criteria have been used for assessing the seismic vulnerability. However, in spite of their wide conceptual scope, from an application point of view even those approaches fall short from being truly generic. One has thus to conclude that an operationl adaptation and transfer of available models to new application domains is extremely limited due to methodological constraints. A generic framework for vulnerability analysis thus requires a substantial amount of conceptual standardization and development. The pivotal part of such a framework is probably the interface definition, i.e., the specification of the inputs needed and the output provided. This concept outlined below only addresses the input side, i.e. it is investigated what a suitable characterization of the hazard impact could look like, but not what the descriptors of the vulnerability (often referred to as damage indicators) are.

In a first step, it is useful that the term vulnerability is defined in as generic a way as possible. For this purpose, the state of a given target object can be described by a vector

$\boldsymbol{S}=\left(s_{1}, s_{2}, \ldots s_{n}\right)$

whose components represent the object's performance characteristics. For example, a residental house can be described by performance characteristics such as "total inhabitable footage", "number of inhabitable rooms", "market value", while a road can be characterized by "available number of lanes", "maximum safe speed", "maximum admissible load". The characteristics are object-specific and have to be selected according to to object's purpose. Initially, they are normalized such that the fully functional and structurally undamaged reference state is defined by

$S_{N D}=\left(s_{1}=1, s_{2}=1, \ldots s_{n}=1\right)$

and the total loss of performance by

$\boldsymbol{S}_{T D}=\left(s_{1}=0, s_{2}=0, \ldots s_{n}=0\right)$

The next step in the concept is the investigation of the way that changes in the performance characteristics can occur, i.e. what "states" $\sigma_{1, \ldots, m}$ of the target or its environment lead to a change in performance. This can be done on an abstract 
Table 1. States that affect the performance characteristics of a road (example).

\begin{tabular}{ll}
\hline Performace characteristics $s$ & States $\sigma$ affecting performance \\
\hline Available number of lanes & Obstacles \\
& Geometric discontinuities \\
& Closure of lanes \\
Maximum safe speed & Geometric discontinuities \\
& Visibility \\
& Road condition (moisture etc.) \\
Maximum admissible load & Deterioration of bridges \\
& Gradient \\
& Subsoil conditions (CBR etc.) \\
\hline
\end{tabular}

level, without any direct reference to a natural hazard. In the above example of the road, an excerpt of the results of this step is shown in Table 1.

In the next conceptual step, the relevant have to be related to impacts associated with natural hazards, i.e. it must be determined what impacts can lead to a certain state or change in state. In other words, causal chains impact $\rightarrow$ state $\rightarrow$ performance are to be identified.

NB: It may seem that the link impact - performance could be made without referring to the intermediate state. The main purpose of introducing the state in the new concept is to reduce the probability of important failure modes not being considered, comparable to failure-mode based risk assessment methods used in technical applications. For model application purposes, the state layer can still be "hidden" by re-integrating it into the performance characterization.

The result of this step is a description of the hazard's impact $I$ using a limited set of components

$\boldsymbol{I}=\left(i_{1}, i_{2}, \ldots i_{m}\right)$

that can be related to the value of performance characteristics $s_{i}$ and represented as functions $\Delta s_{j}=f\left(i_{1}, i_{2}, \ldots i_{m}\right)$ (implicitly including the state of the target or its environment). This mapping is shown in Fig. 1.

The suggested concept is based on the assumption that there exists a minimal set $\boldsymbol{I}_{\min }$ applicable to all arbitrary objects. This means that it is possible to define the $i_{1}, i_{2}, \ldots, i_{k \leq m}$ on the left side in Fig. 1 in a way that it does not need to be changed regardless of how the states and performance characteristics are defined. This $I_{\min }$ represents the generic impact description concept.

Using this approach, the vulnerability of a given target object can now be expressed using the partial differential

$V=f\left(\frac{\partial \boldsymbol{S}}{\partial i_{l}}\right) \quad \forall l \in 1,2, \ldots, k$

i.e. as the relative change in performance that results from a change in the impact.

Conceptually, this definition is not only applicable for physical or structural, but also for "softer" ones such as social or institutional properties. However, it is much more

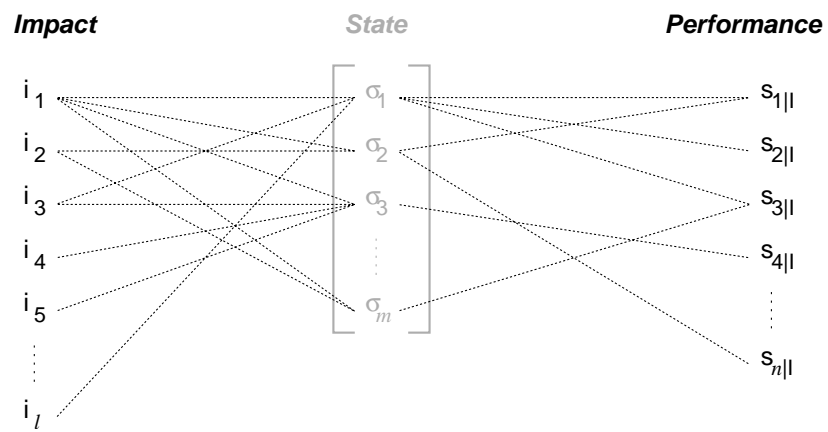

Fig. 1. The concept of mapping impact descriptors to states and states to performance characteristics.

demanding to define performance characteristics and, consequently, states for representing these properties. For an initial implementation, the suggested concept should thus prefearbly be limited to physical vulnerability assessments.

\section{Composition, characteristics and implementation of the generic impact description concept}

4.1 Composition of the generic impact description and its characteristics

Conceptually, the generic impact description interface must comprise elements for all the impacts that can potentially affect the states of any arbitrarily chosen target object, but avoid redundancies whenever possible (both for preventing double-counting and for reducing the effort required for its application). Many of the impacts can be expressed using various terms (e.g., pressure and acceleration can be expressed as force), and consequently, the selection of the descriptors is based on engineering judgement and cannot be unambigously derived from scientific principles. Table 2 contains a preliminary list of components that the author suggests should be part of a generic impact description interface.

Most of the components are well-defined physical variables with internationally agreed-upon (mostly SI) units. In some cases (e.g., for comparisons across a wide range of scales), it may be helpful to refer to a non-parametric formulation of the components.

Both for comparisons and for design and modeling purposes, the components listed in Table 2 must be distinguished in space and time, i.e. in principle the values of

$\boldsymbol{I}=I(t, x, y, z)$

$\boldsymbol{I}_{l}^{\prime}=\frac{d \boldsymbol{I}}{d l_{l \in\{x, y, z\}}}$

$\boldsymbol{I}_{t}^{\prime}=\frac{d \boldsymbol{I}}{d t}$

must ideally be accessible to provide an accurate assessment of the expected spatiotemporal distribution of damage. 
Table 2. Preliminary conceptual list of parameters to be contained in a generic impact description interface.

\begin{tabular}{ll}
\hline Component & $\begin{array}{l}\text { Description } \\
\text { (Type, indicator for) }\end{array}$ \\
\hline $\begin{array}{l}\text { Mechanical impacts } \\
\text { acceleration }\end{array}$ & $\begin{array}{l}\text { vector, external acceleration acting on target } \\
\text { force }\end{array}$ \\
vector, external force acting on target \\
shear stress & number, pressure acting on target surface \\
pulse & numer, shear stress acting on target surface \\
Thermal effects & vector, external pulse acting on target \\
Temperature change & number, change in ambient temperature \\
Ignition potential & boolean, release of ignition sources or fuel \\
Electromagnetic impacts & \\
Changes in conductivity & number, change in ambient conductivity \\
Electromagnetic fields & vector, ambient electromagnetic fields \\
Electrical currents & number, electrical currents \\
$\begin{array}{l}\text { Chemical impacts } \\
\text { Changes in acidity }\end{array}$ & number, change in ambient pH \\
Toxicity & number, toxicity (e.g., relative to $L D_{50}$ ) \\
Changes in $\mathrm{O}_{2}$ & number, change in oxygen level \\
Other impacts & \\
Change in visibility & boolean, optical disturbance by smoke, particles \\
Generation of flying debris & boolean, potential for generating missiles \\
\hline &
\end{tabular}

At a first glance, the requirements for the new concept of a standardized impact description seem much more demanding than those of existing hazard and vulnerability models. These models often work with outputs or inputs that are much less complex, sometimes comprising just one parameter (e.g., the intensity in the case of earthquake-related models). However, the new concept does not aim at replacing simple and proven models, but at complementing and extending their area of application. Conceptually, the output of in most cases, this can be achieved by operations as simple as setting irrelevant parameters to zero and transforming others to those compatible with the generic impact description (e.g., by providing a relation between earthquake intensity and acceleration, see below). The latter case is illustrated in Fig. 2 .

4.2 Concept for the potential implementation of the generic impact description

A generic impact description will ideally be implemented as an interface specification within modularized risk assessment procedures that separate hazard and damage assessment tasks. Adhering to such standard interfaces facilitates both the re-use of existing knowledge (e.g., existing hazard assessment models) and the development of widely applicable hazard and vulnerability models in the future. A modularized and standardized procedure will also be a basis for comparing different models for the same purpose (e.g., applying different vulnerability models for the same target object or different models for the same hazard) and thus for calibration and optimization between computational effort and accuracy.

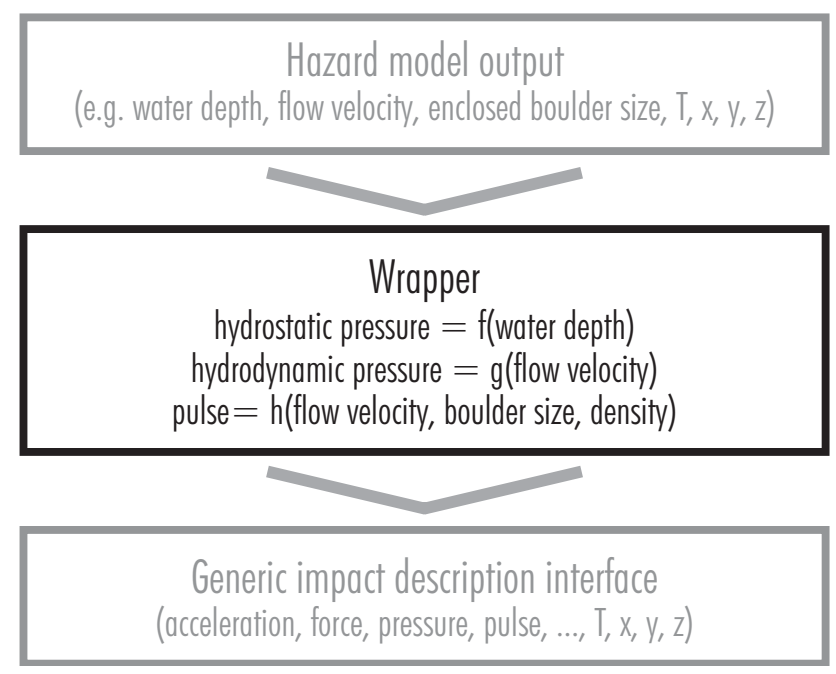

Fig. 2. Wrappers can be used to transform the output of existing hazard assessment models into a form compliant with the generic hazard impact description interface.

Another promising field for implementing the concept of a generic impact description as an interface specification are (self-)learning expert and decision-support systems. These knowledge-based systems will become more important both for classical pre-event risk assessments and for emergency management purposes. Data and computational facilities are increasingly available, but these resources can be utilized to their full potential only if the compatibility of hazard model outputs with vulnerability model inputs is ensured by means of standardization.

\subsection{Potential problems of the concept and its implementa- tion}

As promising as the concept of a generic impact description interface for combining the hazard and vulnerability assessments looks to the author, there are some problems that could make its application difficult.

The first problem - and the most likely to arise - is the acceptance. In Switzerland, risk-based approaches to dealing with natural hazards have been promoted for almost two decades, and only now they are becoming adopted on a widespread basis. This is despite the broad agreement that the underlying concepts are useful for improving the efficiency protective measures. However, the need to re-think proven and well-established practices in favor of new ones that are perceived as competing and more complex has led to a certain reluctance in adopting the new concept. Shortcomings in communicating the benefits of risk-based approaches have probably aggravated the situation. Even today, hazard assessments are still the best accepted subtask within risk assessments for natural hazards; and this is probably true not only for the practice, but for research and development as well. A new concept introduced in such a situation is perhaps perceived as yet another complication of something that just 
Table 3. Beaufort scale and Saffir-Simpson hurricane scale converted to windspeed.

\begin{tabular}{lcccccccc}
\hline $\begin{array}{l}\text { Beaufort } \\
\text { Saffir-Simpson }\end{array}$ & 9 & 10 & 11 & $\begin{array}{c}12 \\
1\end{array}$ & 2 & 3 & 4 & 5 \\
\hline$v\left(\mathrm{~ms}^{-1}\right)$ & $21-25$ & -29 & -33 & -42 & -49 & -58 & -69 & $>69$ \\
\hline
\end{tabular}

started to gain acceptance, and reactions ranging from scepticism to outright rejection might not be surprising. If risk assessments were performed by or under the lead of just one institution, this might give it the authority to implement such a new concept by simply declaring it as mandatory. However, in reality most risk assessments are the collaborative work of several institutions of which none has the competency to define the procedures that the others must apply.

Another issue is the effort required to adapt existing models to the generic interface. Many of them are designed for discrete space and time application and thus not readily available for transformation into a spatiotemporally continuous representation. In other cases, it may be difficult to find appropriate conversions between the output of the model and the components of the generic interface. These are more general problems of the existing models per se: discrete models will never be able to provide truly continuous information, and models outputs that cannot be converted are likely to be in a highly specific form (e.g., with regard to the units they use) and thus never be comparable to other assessment results. However, the adaptation of existing models is certainly a difficulty that must not be underestimated in the concept.

\section{Conceptual application}

The generic impact description interface is still in the conceptual and early prototyping stage. There are yet no case studies available that illustrate the procedure and give an estimate about the cost and benefits of the concept. Instead, the author will show one potential application and various examples of wrappers for existing models.

5.1 The generic impact description interface as a guideline for hazard mapping

Regional-scale hazard mapping is one of the first steps in a comprehensive risk assessment procedures as required by the Swiss forest law Swiss Conf. (1991a) and the law on hydraulic engineering Swiss Conf. (1991b). In the course of the mapping, various hazards are usually investigated, ranging from snow avalanches over torrent-related hazards to landslides. The models used for the hazards differ not only in complexity and accuracy, but also in their output parameters.

The current approach for comparing the impacts of the various hazards is the delineation of zones according to a common scheme. Usually, the following zones are distinguished with regard to the intensity of impacts (the frequency is another criterion, but not considered here):
- Red Zone: The impacts are such that people in buildings are may suffer serious injury or death; buildings may be severely damaged or destroyed.

- Blue Zone: The impacts can seriously injure or kill people that are outdoors while those inside are sufficiently protected; buildings may be damaged, but not to a degree that could result in structural collapse.

The zoning concept is similar to the idea behind the generic interface: one tries to illustrate the impacts of different hazards on a common scale. However, most guidelines define the zones in terms of physical parameters (e.g., flow velocity, flow depth, pressure). Instead of hiding this explicit values behind color codes, the author suggests that they should be transformed in a way that makes them directly comparable using the components of the generic impact description interface. It is planned to apply this idea in a pilot case study in practice, where hazard maps for a region are developed in the traditional way. The focus of the project lies on the procedures and efforts that are necessary to derive comparable and standardized hazard description.

\subsection{Example wrapper definitions}

The following examples illustrate the wrapper concept using a couple of very simple examples. The models used in practical applications are often not much more sophisticated than those shown below, the difference to the "real world" might thus not be very big. One limitation has to be made: the wrappers are not able to provide the time history of the impacts (unless already known from the original hazard model). This is not primarily a proof of the concept's inadequacy, but of the pragmatism that is inherent in most practical models.

\subsubsection{Example wind model wrapper}

From the classification of storms using scales such as the Beaufort or the Saffir-Simpson scale, wind speeds can be derived (see Table 3), and the speed can then be further transformed into force and pressure terms using the relations

$F_{\text {flow }}=\frac{1}{2} c_{w} A \rho v^{2}$

for the turbulent flow friction force and

$p_{\text {flow }}=\frac{1}{2} \rho v^{2}$

for the flow pressure. The other relevant components of the interface such as shear stress (surface erosion), changes in visibility and debris generation will usually also depend on the speed and can be derived using expert judgment. 
Table 4. Earthquake intensity (MMI) to peak ground acceleration (PGA) relation as used in FEMA (1999).

\begin{tabular}{cccccccc}
\hline MMI & VI & VII & VIII & IX & X & XI & XII \\
\hline PGA & 0.12 & 0.21 & 0.36 & 0.53 & 0.71 & 0.86 & 1.15 \\
\hline
\end{tabular}

\subsubsection{Example earthquake model wrapper}

For many areas, earthquake hazard maps are available that indicate the expected intensity (usually related to a certain probability) that results from quakes along known fault systems. The intensity scales such as MSK, MMI or EMS are phenological ones, i.e. they use the damage characterization as measurement criterion. However, there are conversions available between intensity and various physical descriptors of an earthquake. One example is the MMI to PGA relation shown in Table 4.

Using the information in Table 4 as a wrapper, the earthquake impact can now be described using the generic interface: The acceleration is derived from Table 4 , the force, pressure, pulse and temperature components are set to zero, the ignition potential to true, and all the other components to zero or false.

\subsubsection{Example flood model wrapper}

Flood maps are currently available for many areas close to rivers, and they are usually based on hydraulic models. In their simplest form, they only delineate the flooded area, but more sophisticated approaches include water depth and flow velocity. A wrapper for these models is easily designed using the formulas

$p_{\text {stat }}=\rho \times h$

for hydrostatic and Eq. (13) for hydrodynamic pressure. The pulse component can be calculated according to

$p=m \times v$

i.e., basic mechanics play a very central role in the wrapper concept. In the case of floods, the shear stress (channel erosion) and changes in conductivity can also become relevant, and depending on the water depth also the changes in $\mathrm{O}_{2}$. The flood model wrapper is also illustrated in Fig. 2.

\section{Conclusions}

To exploit the full potential of comprehensive risk assessments for natural hazards, the progress in hazard analysis that has been made over the last two decades must be matched by a similar progress in vulnerability analysis. For this purpose, the applicability of the models that are currently available, but also those that will be developed in the future, must be maximized. The suggested concept of standardizing the interface between the hazard model output and the vulnerability model input is one step in this direction.
What remains to be done is the validation of the concept by applying a prototype of the interface description to the requirements of a real-world situation. Once this has been achieved, the implementation of the standard interface in expert and decision support systems could be a useful tool both for promoting the idea and for illustrating its applicability and benefits.

Edited by: T. Glade

Reviewed by: two referees

\section{References}

ATC: Earthquake Damage Evaluation Data for California, Tech. Rep. ATC-13, Applied Technology Council, 1985.

Balendra, T., Tan, K. H., and Kong, S. K.: Vulnerability of Reinforced Concrete Frames in Low Seismic Region, when Designed According to BS 8110, Earthquake Engineering and Structural Dynamics, 28, 1361-1381, 1999.

Coburn, A. and Spence, R.: Earthquake Protection, Wiley, 1992.

Dameron, R. A. and Parker, D. R.: Seismic analysis of concrete towers of the San Diego-Coronado Bridge and evaluation of a high performance concrete retrofit, in: Worldwide Advances in Structural Concrete and Masonry, 1996.

Dowrick, D. J. and Rhoades, D. A.: Vulnerability of different classes of low-rise buildings in the 1987 Edgecumbe, New Zealand, earthquake, Bulletin of the New Zealand National Society for Earthquake Engineering, 30, 227-241, 1997.

Dean, E. T. and Soulage, J. R.: Using Wind Design Criteria to Gauge Seismic Performance, in Structural Engineering in the 21st Century, Proceedings of the 1999 Structures Congress, ASCE, 1999

FEMA: HAZUS99 SR2 Technical Manual, Federal Emergency Management Agency, Washiongton, D.C., 1999.

Hollenstein, K., Bieri, O., and Stückelberger, J.: Modellierung der Vulnerability von Schadenobjekten gegenüber Naturgefahrenprozessen, Online publication, http://e-collection.ethbib.ethz.ch/ show?type=bericht $\backslash \& n r=173$, ETHZ Forest Engineering, 2002.

IUGS: Quantitative risk assessment for slopes and landslides - The state of the art, pp. 3-12, A.A. Balkema, Rotterdam/Brookfield, proceedings of the international workshop on landslide risk assessment, Honolulu, Hawaii, USA, 19-21 February 1997.

Porter, K. A., Kiremidjian, A. S., and LeGrue, J. S.: AssemblyBased Vulnerability of Buildings and Its Use in Performance Evaluation, Earthquake Spectra, 17, 291-312, 2001.

Swiss Conf.: Forest Law, SR 921, 1991a.

Swiss Conf.: Law on Hyrdaulic Engineering, SR 721, 1991b. 